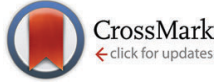

Cite this: DOI: $10.1039 / \mathrm{c} 5 \mathrm{mb} 00281 \mathrm{~h}$

\title{
Probing the epigenetic regulation of HIF-1a transcription in developing tissue
}

\author{
I. N. Mistry, ${ }^{a}$ P. J. S. Smith, ${ }^{b}$ D. I. Wilson ${ }^{c}$ and A. Tavassoli*ab
}

HIF-1 is the master regulator of cellular hypoxia response; the oxygen sensitive HIF-1 $\alpha$ subunit transactivates its own expression in hypoxia via a hypoxia response element (HRE) in the promoter of the HIF-1 $\alpha$ gene. This transactivation loop significantly contributes to the build up of HIF-1 $\alpha$ at the onset of hypoxia, with the binding of HIF-1 to the HIF-1 $\alpha$ promoter being dependent on the epigenetic status of a $\mathrm{CPG}$ dinucleotide in the upstream HRE. Given the central role played by HIF-1 in tissue development, we sought to probe the epigenetic status of the HIF-1 $\alpha$ HRE and that of its downstream target EPO in embryonic tissue. Our data shows that the CPG dinucleotide in HIF-1 $\alpha$ HRE is unmethylated in several embryonic tissue samples, suggesting that transactivation of HIF-1 $\alpha$ plays a significant role in HIF-1 mediated hypoxia response during development.

www.rsc.org/molecularbiosystems

HIF-signalling plays a significant role. HIF co-ordinates the development of the blood, vasculature and nervous system, and affects the morphogenesis of the embryo and placenta. ${ }^{13,14}$

HIF- $1 \alpha$ expression and activity is controlled at the transcription, translation and post-translation levels, and there is increasing evidence that epigenetic changes, particularly CpG methylation, are also key determinants of hypoxia-response. The methylation state of a CpG dinucleotide in the upstream promoter of HIF-1 $\alpha$ for example, has been recently found to correlate with the rate of HIF- $1 \alpha$ accumulation in hypoxic cancer cells. ${ }^{15,16}$ This is due to the presence of an HRE within a CpG island in the promoter region of the HIF-1 $\alpha$ gene (ACGTG, 156 bases upstream from the transcription start site), ${ }^{7}$ which results in the transactivation of HIF- $1 \alpha$ transcription by the HIF-1 transcription factor at the onset of hypoxia (Fig. 1A). ${ }^{15,16}$ This results in a significantly accelerated rise in HIF- $1 \alpha$ at both the mRNA and protein level, which in turn increases the transcription of other HIF-1 target genes; methylation of this CpG dinucleotide has been shown to inhibit HIF-1 binding (in vitro and in cells). ${ }^{15,16}$ The HRE-dependent transcription of several HIF-1 target genes (e.g. erythropoietin (EPO) and glucose transporter 3 ) has also been shown to be regulated by a similar mechanism, ${ }^{17,18}$ suggesting a wider role for epigenetic control of HIF-mediated transcription in hypoxia. The epigenetic status of the HIF- $1 \alpha$ HRE in various cell and tissue types has been little explored, despite its potential significance for determining the extent of hypoxia response. A recent study has found HIF- $1 \alpha$ mRNA expression to be exceptionally low in HMC-1 cells, a model immature mast cell line. ${ }^{19}$ In vitro methylation of the HIF- $1 \alpha$ promoter caused a significant decrease in reporter gene expression whereas treatment with the DNA demethylating agent $5^{\prime}$-azacytidine resulted in a dose dependent increase in expression of the gene, suggesting the low expression of HIF- $1 \alpha$ in the HMC- 1 cells was caused by a 
A

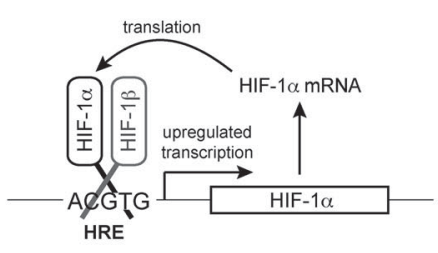

C

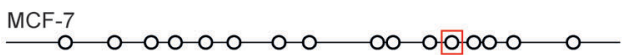

B

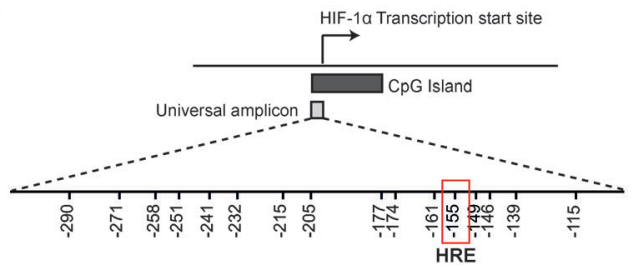

HCT-116

Fig. 1 Analysis of the methylation status of the HIF-1 $\alpha$ promoter via bisulphite sequencing. (A) The HIF-1 $1 \alpha$ transactivation loop. (B) Graphical representation of the position of the region of the HIF-1 $\alpha$ promoter analysed and the $16 \mathrm{CpG}$ sites within this region. Numbers represent position (in base pairs) relative to the HIF-1 $\alpha$ TSS. (C) Methylation status of this region in DNA extracted from MCF7 and HCT116 cell lines. Methylation status of each CpG site is represented by circles, where white is unmethylated and black is methylated. He CpG situated in the HIF-1 $\alpha$ HRE is highlighted.

methylated HIF-1 $\alpha$ promoter. ${ }^{19}$ Given the important role played by HIF-1 in embryonic development, and the potential significance of the HIF- $1 \alpha$ transactivation loop in controlling hypoxia response, we hypothesised that the need for rapid hypoxia response in developing tissue would require an unmethylated HIF-1 $\alpha$ HRE. As the default epigenetic status of the HIF- $1 \alpha$ HRE during various stages of development is currently unknown, we sought to assess the methylation status of the HIF-1 $\alpha$ HRE in developing embryonic tissue.

\section{Results and discussion}

\section{The HIF-1 $\alpha$ HRE is unmethylated in embryonic DNA}

Primers were designed, using MethPrimer software, ${ }^{20}$ to amplify the promoter region of HIF- $1 \alpha$ from bisulfite-treated chromosomal DNA, regardless of methylation status. We began by validating our epigenetic sequencing method using DNA extracted from cancer cell lines. MCF-7 breast cancer cells, which are known to have an unmethylated HIF- $\alpha$ HRE, ${ }^{21}$ were used as a negative control. HCT-116 colon cancer cells, which are known to have a methylated HIF- $1 \alpha$ HRE, were used as a positive control. ${ }^{16}$ A $220 \mathrm{bp}$ region of the HIF-1 $\alpha$ promoter containing $16 \mathrm{CpG}$ sites (Fig. 1B), including the one within the HIF-1 $\alpha$ HRE (ACGTG) was analysed by bisulphite sequencing. The sequencing data verified that the cytosine residues in CpG dinucleotides in the HIF- $1 \alpha$ promoter region, and particularly the cytosine residue in the HIF- $1 \alpha$ HRE, were unmethylated in MCF7s, and methylated in HCT-116s (Fig. 1C).

We next applied this technique to embryonic DNA, isolated from 7 tissue types (bowel, brain, kidney, stomach, muscle, adrenal, lung or tongue). These samples were from 4 embryos 55-59 days post conception. No major epigenetic events are known to take place in this period of development, ${ }^{22}$ allowing a comparable view of the methylation status of the HIF- $1 \alpha$ promoter. The methylation status of these DNA samples was determined by bisulfite sequencing with the above primers.

We found the CpG island region of the HIF- $1 \alpha$ promoter to be largely unmethylated (Fig. 2A). We observed that on average, $1.1 \%$ of CpGs were methylated with no more than $2 \%$ methylation in each tissue (Fig. 2C). There was greater variation in the proportion of methylated CpGs at each position of the HIF- $1 \alpha$ promoter
(Fig. 2B), with a maximum of $3.8 \%$ methylation at position $-146 \mathrm{bp}$ and $3.1 \%$ methylation at position $-241 \mathrm{bp}$ (both relative to the HIF- $1 \alpha$ TSS). However large errors on these values show that methylation percentages at these positions are likely being positively skewed by few highly methylated samples, and that they are not significantly different to unmethylated positions.

The CpG site in the HIF- $1 \alpha$ HRE, 155 bp upstream of the HIF- $1 \alpha$ transcription start site (Fig. 1B and 2A, boxed), was unmethylated in $99 \%$ of clones sequenced. Histoimmunochemical analysis of mouse embryos, using the hypoxia marker pimonidazole and its associated antibody, have shown that hypoxic cells are widespread in the early stages of embryo development, and that these hypoxic regions co-localize with HIF expression. ${ }^{23,24}$ This data suggests that the HIF- $1 \alpha$ transactivation loop is active in embryonic tissue, with the HIF-1 transcription factor able to bind to the unmethylated HRE and upregulate expression of HIF- $1 \alpha$. Previous chromatin immunoprecipitation assays with HCT-116 cells have shown that HIF-1 is only recruited to an unmethylated HIF- $1 \alpha$ promoter, and HIF1 does not bind to the HIF- $1 \alpha$ promoter when it is methylated. ${ }^{16}$ Binding of HIF- 1 to the HIF- $1 \alpha$ HRE amplifies the extent of HIF1 mediated hypoxia signaling by upregulating HIF- $1 \alpha$ transcription. The downstream effect of this will be an increase in the rate of hypoxic transcription of HIF-1 target genes, such as those involved in vasculogenesis, erythropoiesis and angiogenesis, by HIF- $1 .{ }^{13}$

\section{Analysis of the methylation status of the EPO HRE in embryonic DNA}

To evaluate whether the unmethylated status of the HIF- $1 \alpha$ promoter was a reflection of global demethylation of HREs, we analysed the methylation status of EPO, another target of HIF-1. The gene encoding EPO resides on chromosome 7, whereas the HIF- $1 \alpha$ gene resides on chromosome 14 . The $3^{\prime}$ enhancer of EPO lacks a CpG island,${ }^{17}$ but previous studies have shown that hypermethylation in the $3^{\prime}$ enhancer region leads to a decrease in EPO expression in primary cancer cell lines, as an unmethylated EPO HRE is required for HIF-1 binding. ${ }^{17,25}$ We therefore determined the methylation status of the $6 \mathrm{CpG}$ dinucleotides in the EPO enhancer element in embryonic DNA samples by bisulphite sequencing (Fig. 3A). It should be noted that the same bisulphite treated DNA used in the HIF- $1 \alpha$ study was used for 
A
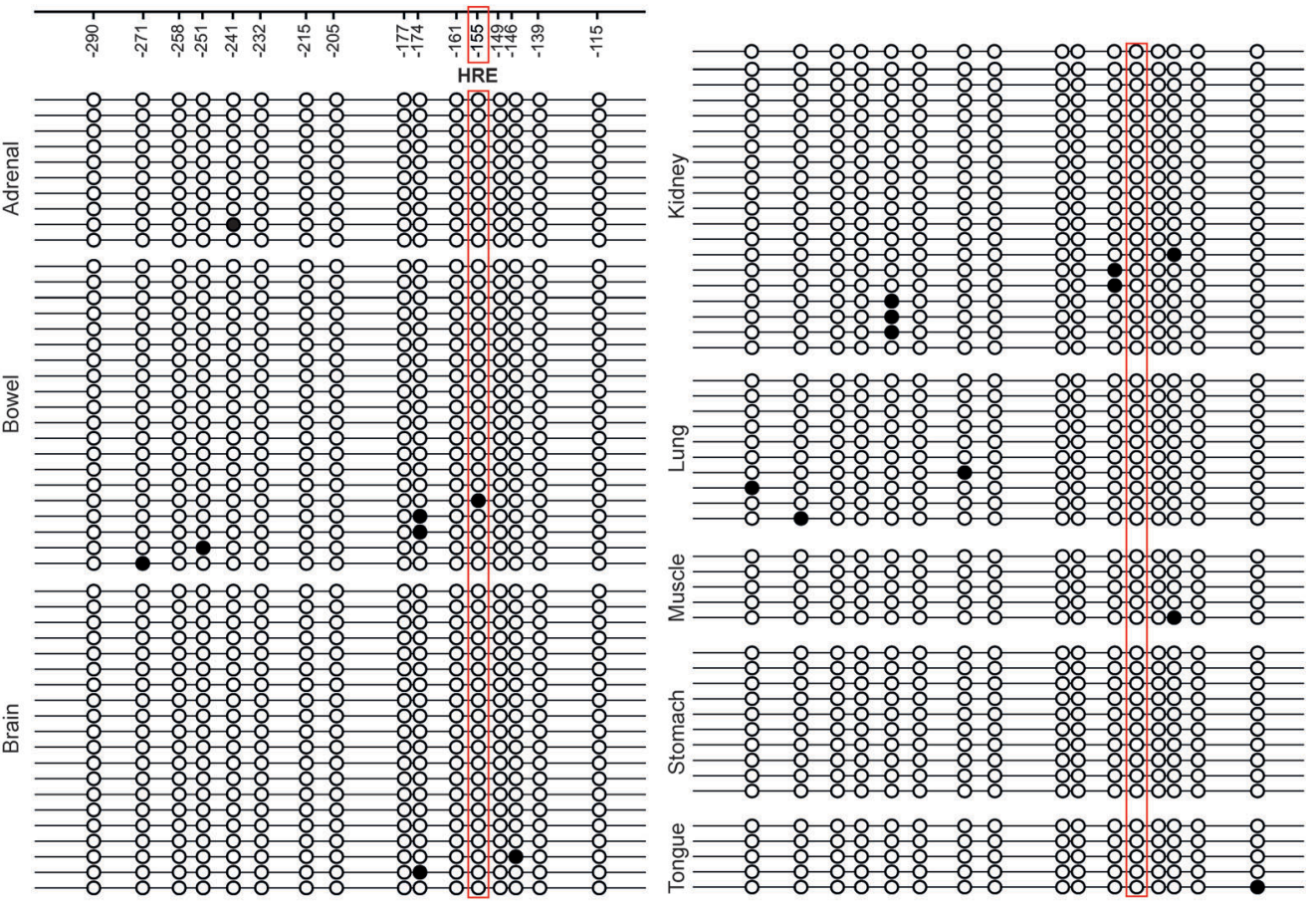

B

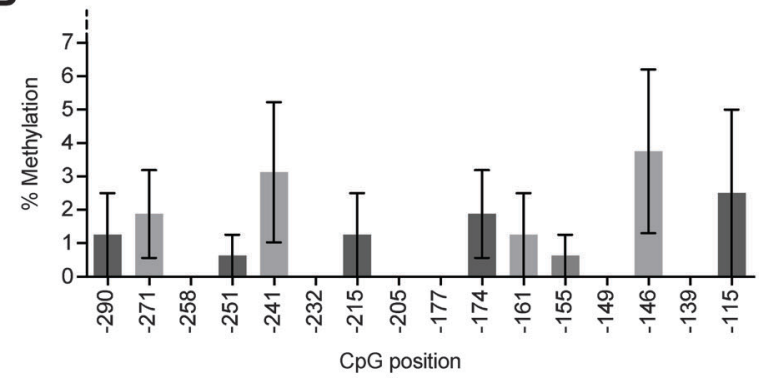

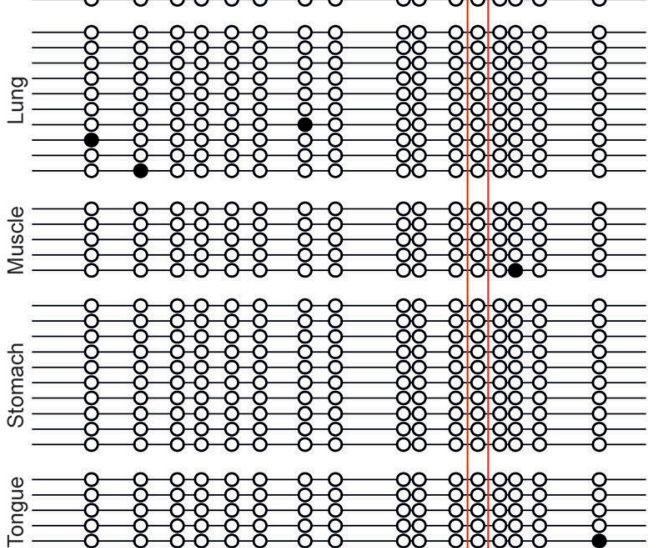

C

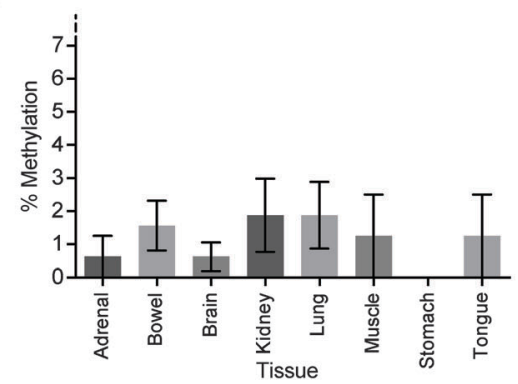

Fig. 2 Analysis of the methylation status of the HIF-1 $\alpha$ HRE in DNA extracted from embryonic tissue. (A) Methylation status of each CpG site represented by circles, where white is unmethylated and black is methylated. The CpG situated in the HIF-1 $\alpha$ HRE is highlighted. Each row represents data from one sequenced clone, which are grouped by tissue: adrenal, bowel, brain, kidney, lung, muscle, stomach and tongue. 5 clones of each DNA sample were sequenced. Scale bar shows position (bp) relative to the HIF-1 $\alpha$ TSS. (B) Percentage methylation of above CpG sites grouped by CpG position, bars represent mean proportion of methylated clones at each position \pm SEM. (C) Percentage methylation of above CpG sites by tissue, bars represent mean proportion of methylated clones in each tissue \pm SEM.

evaluation of EPO to ensure consistency between the analysis of the two genes.

In contrast to the HIF- $1 \alpha$ promoter, we observed a high degree of methylation in the EPO enhancer, with $47 \%$ methylation across all CpG sites (Fig. 3A). It may therefore be concluded that the unmethylated state of the HIF- $1 \alpha$ promoter is not indicative of global demethylation, but a result of specific epigenetic regulation in that region. Interestingly, there were significant differences (ranging between $21 \%$ and $63 \%$ ) in the methylation state of individual CpG sites within the EPO enhancer for each tissue, and between tissues (Fig. 3B). The CpG position situated within the HRE of EPO had the lowest average methylation in the locus studied; only $21 \%$, compared to the average $47 \%$, this value was significantly lower than other EPO CpG sites, except for the site adjacent to the EPO HRE (97 bp downstream from the EPO $3^{\prime}$ polyadenylation site). The relative lack of methylation of the CpG site in the EPO HRE indicates that despite methylation of the enhancer region, binding of HIF-1 and transcriptional activation of EPO can still occur in these tissues. Previous studies have shown that methylation of the CpG residue within the core HRE (ACGTG) alone prevents binding of HIF-1 to the EPO enhancer region, ${ }^{17}$ indicating the importance of HRE DNA methylation in dictating HIF-1 association with DNA. The level of methylation in the EPO enhancer varied between tissues from $29 \%$ in tongue to $65 \%$ in stomach (Fig. 3C). This disparity between tissues may be a result of differing degrees of hypoxia throughout the developing embryo. For example, areas of low oxygen are more consistently detected in specific regions of the embryo such as the developing heart, gut and skeleton. ${ }^{13,26}$ This is likely due the significant growth and energy demands of these tissues. ${ }^{13}$ While the molecular basis of 
A

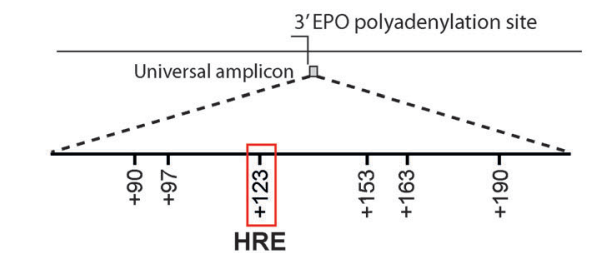

B
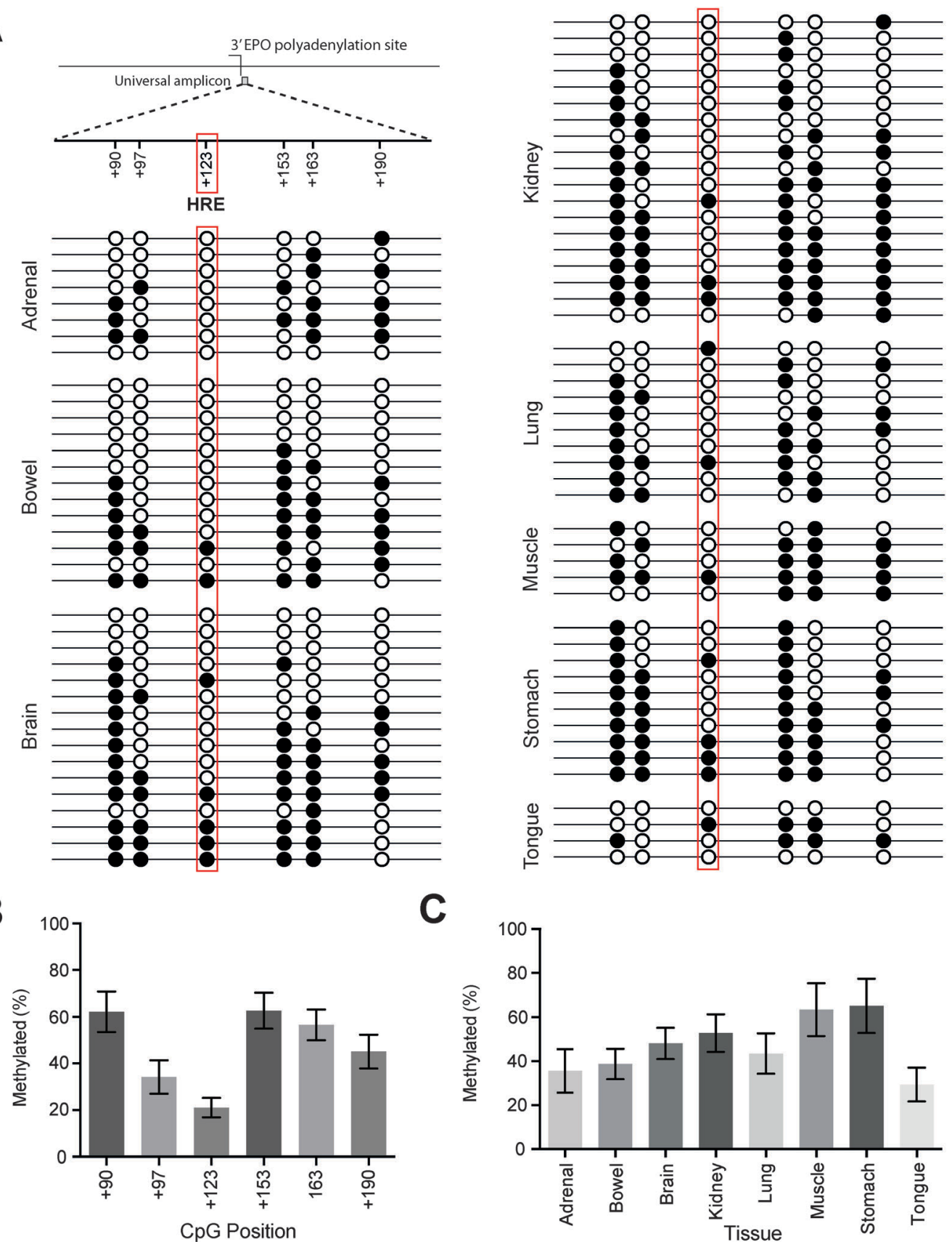

C

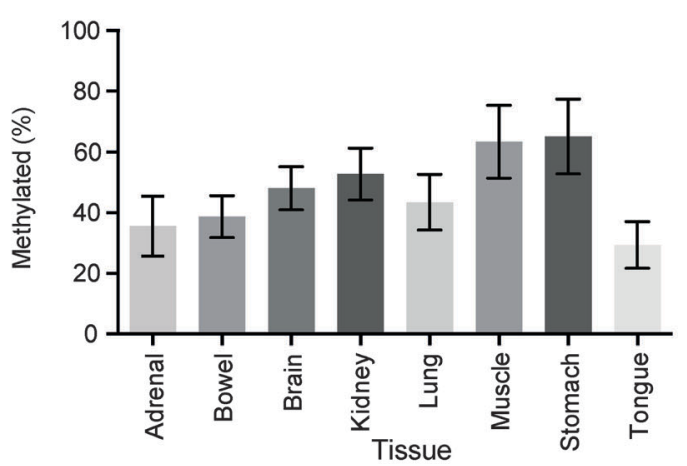

Fig. 3 Analysis of the methylation status of the EPO enhancer element by bisulphite sequencing. (A) Graphical representation of the region of the EPO enhancer analysed, and the $6 \mathrm{CpG}$ sites within this region followed by the methylation status of each CpG site represented by circles, where white is unmethylated and black is methylated. The CpG situated in the EPO HRE is highlighted. Each row represents data from one sequenced clone, which are grouped by tissue: adrenal, bowel, brain, kidney, lung, muscle, stomach and tongue; 5 clones of each DNA sample were sequenced. Scale bar shows position (bp) relative to the EPO 3' polyadenylation site. (B) Percentage methylation of all CpG sites grouped by CpG position, bars represent mean proportion of methylated clones at each position \pm SEM. (C) Percentage methylation of all CpG sites grouped by tissue, bars represent mean proportion of methylated clones in each tissue \pm SEM.

such selective demethylation events are not clear, the increased methylation in the region as a whole may dampen transcriptional activation by recruiting methyl binding proteins and histone deacetylases, exemplifying the complexity of the interlinking epigenetic mechanisms of gene regulation. ${ }^{25}$

Comparison of the above with the methylation status of the HIF-1 $\alpha$ HRE in normal adult tissue would allow a better understanding of the relevance of HIF- $1 \alpha$ HRE demethylation and the role of the HIF- $1 \alpha$ transactivation loop during development. However, only a few examples of such data could be found in the literature. The HIF- $1 \alpha$ promoter has been shown to be unmethylated in histopathologically unchanged colon tissue ${ }^{27}$ and normal uterine cervical tissue. ${ }^{28}$ Interestingly, both these studies revealed that, despite the presence of an unmethylated HRE, HIF- $1 \alpha$ mRNA and 
protein expression was low in these normal tissues and upregulated in equivalent cancerous tissue. ${ }^{27,28}$ These findings underline the importance of post-translational regulation in regulating intracellular HIF- $1 \alpha$ levels, and suggest that there may be selective pressure for the HRE CpG site to remain unmethylated to enable more rapid recovery of oxygen homeostasis at the onset of hypoxia, independently of HIF- $1 \alpha$ expression. Selective pressure for an unmethylated HRE independent of gene expression levels was similarly suggested for the EPO HRE, where hypomethylation of the EPO HRE was found in human cell lines and mouse tissues that do not express EPO. $^{17}$

\section{Conclusions}

There is increasing evidence that HIF- $1 \alpha$ expression varies in different tissues and cell types, and that this variation may be mediated by DNA methylation of the gene promoter. ${ }^{15,16,19}$ Our data elucidates the unmethylated status of the HIF- $1 \alpha$ HRE in embryonic DNA samples from various tissues, indicating that this gene is available for rapid transactivation in the hypoxic conditions of embryonic development. In addition, we show that the enhancer region of EPO has a varying degree of methylation between tissues but a consistently low level of methylation at the CpG site within its HRE, suggesting additional regulation of EPO expression. These results give insight into the transcriptional regulation of HIF- $1 \alpha$ and its target EPO in developing tissue.

\section{Experimental}

\section{Cell culture}

MCF-7 breast carcinoma cells were maintained in DMEM (Life Technologies) containing 10\% FBS. HCT-116 colon carcinoma cells were maintained in Mcoys's 5A medium (Life Technologies) containing $10 \%$ FBS. Both cell lines were cultured at $37{ }^{\circ} \mathrm{C}$ in $5 \%$ $\mathrm{CO}_{2}$.

\section{Extraction of DNA from cancer cell lines}

Confluent MCF-7 and HCT-116 cells were collected by trypinisation and the cell pellet washed with PBS. The cells were lysed and genomic DNA extracted using GenElute Mammalian Genomic DNA miniprep kit (Sigma Aldrich).

\section{Tissue collection and DNA extraction}

Embryonic tissue was obtained from women undergoing termination of pregnancy with written informed consent and ethical approval. (Southampton \& South West Hampshire Local Research Ethics Committee 296/00). Developmental staging was determined by foot length and Carnegie staging. DNA was extracted from tissues as previously described. ${ }^{29}$

\section{Bisulphite sequencing}

Extracted DNA was treated with sodium bisulphite under basic conditions with Imprint DNA modification kit (Sigma Aldrich) and the region of interested amplified with universal primers $5^{\prime}$-tgatgtatgtttgggattaggtaat- 3 and $5^{\prime}$-ctctcaaccaatcaaaaaac- $3^{\prime}$ for
HIF- $1 \alpha$ and $5^{\prime}$-tggtagtagtgtagtaggtttaggtt- $3^{\prime}$ and $5^{\prime}$-aaataaaacca ccttattaaccaac- $3^{\prime}$ for EPO with Platinum Taq DNA polymerase (Life Technologies). The amplified sequences were then TA cloned into pGEM-EasyT vectors (Promega), transformed into E. coli. and positive clones were identified by blue/white screening. Five clones of each DNA sample were sequenced.

\section{Data analysis}

The sequencing data file for each clone was aligned and analysed by BiQ analyser. ${ }^{30}$ Sequences with a bisulphite conversion rate of less than 95\% were discounted, as were erroneous sequencing and sequences likely to come from the chromosome of the same cell. Sequences were grouped by tissue or position and the mean proportion of methylated CpGs per 5 clones of the same sample was calculated; unpaired $t$-tests were used to compare mean values and determine statistical significance.

\section{Acknowledgements}

The authors thank the University of Southampton Institute for Life Sciences, and the Engineering and Physical Sciences Research Council (EP/J500537/1) for funding this work (PhD studentship to I.N.M).

\section{Notes and references}

1 G. L. Wang, B. H. Jiang, E. A. Rue and G. L. Semenza, Proc. Natl. Acad. Sci. U. S. A., 1995, 92, 5510-5514.

2 A. C. R. Epstein, J. M. Gleadle, L. A. McNeill, K. S. Hewitson, J. O'Rourke, D. R. Mole, M. Mukherji, E. Metzen, M. I. Wilson, A. Dhanda, Y.-M. Tian, N. Masson, D. L. Hamilton, P. Jaakkola, R. Barstead, J. Hodgkin, P. H. Maxwell, C. W. Pugh, C. J. Schofield and P. J. Ratcliffe, Cell, 2001, 107, 43-54.

3 N. Masson, C. Willam, P. H. Maxwell, C. W. Pugh and P. J. Ratcliffe, EMBO J., 2001, 20, 5197-5206.

4 P. Jaakkola, D. R. Mole, Y. M. Tian, M. I. Wilson, J. Gielbert, S. J. Gaskell, A. von Kriegsheim, H. F. Hebestreit, M. Mukherji, C. J. Schofield, P. H. Maxwell, C. W. Pugh and P. J. Ratcliffe, Science, 2001, 292, 468-472.

5 D. Chilov, G. Camenisch, I. Kvietikova, U. Ziegler, M. Gassmann and R. H. Wenger, J. Cell Sci., 1999, 112, 1203-1212.

6 H. Kimura, A. Weisz, T. Ogura, Y. Hitomi, Y. Kurashima, K. Hashimoto, F. D’Acquisto, M. Makuuchi and H. Esumi, J. Biol. Chem., 2001, 276, 2292-2298.

7 E. Minet, I. Ernest, G. Michel, I. Roland, J. Remacle, M. Raes and C. Michiels, Biochem. Biophys. Res. Commun., 1999, 261, 534-540.

8 B. L. Ebert and H. F. Bunn, Mol. Cell. Biol., 1998, 18, 4089-4096.

9 U. R. Jewell, I. Kvietikova, A. Scheid, C. Bauer, R. H. Wenger and M. Gassmann, FASEB J., 2001, 15, 1312-1314.

10 J. I. Bárdos and M. Ashcroft, Biochim. Biophys. Acta, 2005, 1755, 107-120.

11 A. L. Harris, Nat. Rev. Cancer, 2002, 2, 38-47.

12 G. L. Semenza, Nat. Rev. Cancer, 2003, 3, 721-732. 
13 S. L. Dunwoodie, Dev. Cell, 2009, 17, 755-773.

14 M. C. Simon and B. Keith, Nat. Rev. Mol. Cell Biol., 2008, 9, 285-296.

15 D. J. Asby, F. Cuda, F. Hoakwie, E. Miranda and A. Tavassoli, Mol. BioSyst., 2014, 10, 2505-2508.

16 M. Koslowski, U. Luxemburger, O. Tureci and U. Sahin, Oncogene, 2011, 30, 876-882.

17 R. H. Wenger, I. Kvietikova, A. Rolfs, G. Camenisch and M. Gassmann, Eur. J. Biochem., 1998, 253, 771-777.

18 I. Mimura, M. Nangaku, Y. Kanki, S. Tsutsumi, T. Inoue, T. Kohro, S. Yamamoto, T. Fujita, T. Shimamura, J. Suehiro, A. Taguchi, M. Kobayashi, K. Tanimura, T. Inagaki, T. Tanaka, T. Hamakubo, J. Sakai, H. Aburatani, T. Kodama and Y. Wada, Mol. Cell. Biol., 2012, 32, 3018-3032.

19 A. Walczak-Drzewiecka, M. Ratajewski, L. Pulaski and J. Dastych, Biochem. Biophys. Res. Commun., 2010, 391, 1028-1032.

20 L. C. Li and R. Dahiya, Bioinformatics, 2002, 18, 1427-1431.

21 E. Miranda, I. K. Nordgren, A. L. Male, C. E. Lawrence, F. Hoakwie, F. Cuda, W. Court, K. R. Fox, P. A. Townsend, G. K. Packham, S. A. Eccles and A. Tavassoli, J. Am. Chem. Soc., 2013, 135, 10418-10425.
22 W. Reik, Nature, 2007, 447, 425-432.

23 Y. M. Lee, C. H. Jeong, S. Y. Koo, M. J. Son, H. S. Song, S. K. Bae, J. A. Raleigh, H. Y. Chung, M. A. Yoo and K. W. Kim, Dev. Dyn., 2001, 220, 175-186.

24 K. G. Pringle, K. L. Kind, J. G. Thompson and C. T. Roberts, Placenta, 2007, 28, 1147-1157.

25 K. Steinmann, A. M. Richter and R. H. Dammann, Genes Cancer, 2011, 2, 65-73.

26 M. Ream, A. M. Ray, R. Chandra and D. M. Chikaraishi, Am. J. Physiol.: Regul., Integr. Comp. Physiol., 2008, 295, R583-595.

27 A. A. Rawluszko-Wieczorek, K. Horbacka, P. Krokowicz, M. Misztal and P. P. Jagodzinski, Mol. Cancer Res., 2014, 12, 1112-1127.

28 M. W. Luczak, A. Roszak, P. Pawlik, H. Kedzia, M. Lianeri and P. P. Jagodzinski, Oncol. Rep., 2011, 26, 1259-1264.

29 T. Hearn, G. L. Renforth, C. Spalluto, N. A. Hanley, K. Piper, S. Brickwood, C. White, V. Connolly, J. F. Taylor, I. RussellEggitt, D. Bonneau, M. Walker and D. I. Wilson, Nat. Genet., 2002, 31, 79-83.

30 C. Bock, S. Reither, T. Mikeska, M. Paulsen, J. Walter and T. Lengauer, Bioinformatics, 2005, 21, 4067-4068. 